\title{
Patientenapotheke Ja oder Nein?
}

Sven Bradke

Geschäftsführer der Ärzte mit Patientenapotheke (APA)
Wenn es nach dem Willen von Bundesrat Couchepin ginge, so wäre die Fragestellung «Patientenapotheke Ja oder Nein» bereits entschieden. Er meint, dass die Medikamentenabgabe eine Aufgabe der Apotheker sei und Ärzte es nicht nötig hätten, diese Dienstleistung anzubieten. Geschweige denn, hierfür auch noch Geld zu bekommen. Er werde deshalb beim Revisionsentwurf des Heilmittelgesetzes die Abschaffung der Selbstdispensation vorsehen.

\section{Waren die Zürcher Abstimmungen überflüssig?}

Wer dies liest, der stellt sich unweigerlich die Frage, ob die drei gewonnenen Zürcher Abstimmungen somit unnötig waren. Jetzt, da sich das Zürcher Stimmvolk klar und deutlich für den Slogan «Ärzte und Apotheken sollen Medikamente abgeben dürfen» aussprach, soll die ärztliche Medikamentenabgabe durch einen Bundesentscheid verboten oder eingeschränkt werden? Jetzt, da in 17 Deutschschweizer Kantonen die Selbstdispensation fest verankert ist, soll eine politische Kehrtwende vollzogen werden?

\section{Medikamentenabgabe und Stethoskop}

Die ärztliche Medikamentenabgabe gehört zum Arzt wie das Stethoskop. Man stelle sich vor, die Ärzte dürften weder bei Notfällen noch im Alltag noch am Spitalbett Medikamente abgeben. Hierfür wäre allein der jeweilige Apotheker zuständig. Wer ein solches Arztverständnis an den Tag legt, der hat von der heutigen Medizin wenig verstanden. Medikamente sind ein wichtiger und essentieller Bestandteil einer umfassenden Therapie. Und zwar im Spital ebenso wie in der Arztpraxis. Eine Medizin ohne Medikamente ist heute schlichtweg undenkbar. Medikamente sind ein integraler Bestandteil einer sicheren, wirtschaftlichen, zweckmässigen und ganzheitlichen Therapie geworden. Sie gehören deshalb zum notwendigen Instrumentarium eines jeden Arztes.

Korrespondenz:

APA

Dr. Sven Bradke

Röschstrasse 18

CH-9006 St.Gallen

Tel. 0712465140

Fax 0712465101

info@patientenapotheke.ch

www.patientenapotheke.ch

\section{Führung einer Patientenapotheke}

Wer eine Patientenapotheke führt, der weiss, dass dies mit entsprechendem Aufwand und Einsatz verbunden ist. Alleine die verschiedenen Vorschriften der Kantonsapotheker zur sicheren und fachgerechten Aufbewahrung der Präparate erfordern eine spezielle Organisation. Zudem haben die eingekauften und gelager-

Die Vereinigung der Ärzte mit Patientenapotheke (APA) ist eine Ärzteorganisation, die sich der Erhaltung und Ausweitung der ärztlichen Medikamentenabgabe widmet. Sie führt im weiteren Ausbildungen und Seminare zur korrekten Führung einer Patientenapotheke sowie zu anderen Themen rund um Medikamente durch. Die über 1000 Mitglieder werden mehrmals jährlich über die neusten Entwicklungen im Bereich der Medikamente informiert.

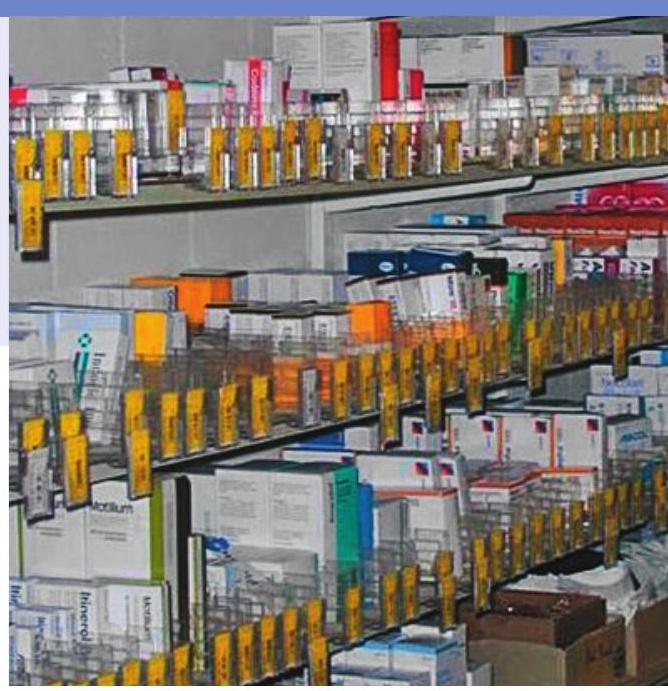

ten Medikamente auch ihren Preis. Am teuersten sind vor allem jene, die dem Patienten wegen einer Unachtsamkeit nicht verrechnet werden ...

\section{Patienten schätzen die ärztliche Medikamentenabgabe}

Dieser Aufwand wiegt aber die Vorteile einer Patientenapotheke in keiner Weise auf. Wer eine solche führt, der kennt die Namen, Logos, Preise und Wirkstoffe der Präparate. Er kennt aus eigener Erfahrung auch die Wirkungen, die möglichen Nebenwirkungen und die entsprechenden Beipackzettel. Im weiteren hat der Arzt die Chance, dem Patienten die Einnahme direkt zu erklären. Aufgrund der Abgabe und der persönlichen Erläuterung in der Praxis kann auch die Compliance wesentlich gesteigert werden. Letztlich bleibt dem Patienten auch der je nach Krankheit äusserst mühsame Weg in die nächste Apotheke erspart. Und zuallerletzt sind die Medikamente beim Arzt in der Regel auch günstiger. Denn hier zahlt der Patient keine Pauschalen, Taxen oder Gebühren, wie sie bei den Apotheken im Rahmen der LOA verlangt werden können.

\section{Dienstleistung am Patienten}

Auch wenn die Margen per 1. Oktober 2009 tiefer liegen als bisher, Medikamente abgeben zu dürfen, ist eine echte Dienstleistung am Kunden. Die Patienten schätzen diese und wollen sie in den bisherigen Abgabekantonen auch weiterhin nutzen dürfen.

\section{Gemeinsam für die SD einstehen!}

Wer bereits heute eine Patientenapotheke führen durfte, der kennt die Vorteile der ärztlichen Medikamentenabgabe. Wer dies bisher noch nicht durfte, der wird es beispielsweise im Kanton Zürich merken, sobald dort die Gesetzesinitiative rechtskräftig wird. Alle Ärzte rufe ich aber auf, national wie kantonal, für die ärztliche Medikamentenabgabe einzustehen und gegen die Angriffe auf Bundesebene anzutreten. Denn wer die ärztliche Medikamentenabgabe angreift, der greift die ärztliche Tätigkeit als Ganzes an. In diesem Sinne danke ich im Namen der Ärzte mit Patientenapotheke (APA) allen, die bei uns Mitglied sind, und hoffe, dass weitere engagierte Kämpfer für die Selbstdispensation zu uns stossen. Denn nur gemeinsam sind wir stark! 